\title{
Genetic polymorphisms of 3'-untranslated region of SULT1A1 and their impact on tamoxifen metabolism and efficacy
}

\author{
A. B. Sanchez-Spitman ${ }^{1,2} \cdot$ V. O. Dezentjé ${ }^{3} \cdot$ J. J. Swen ${ }^{1,2} \cdot$ D. J. A. R. Moes ${ }^{1,2} \cdot$ H. Gelderblom ${ }^{4} \cdot$ Henk-Jan Guchelaar ${ }^{1,2}$
}

Received: 13 June 2018 / Accepted: 12 August 2018 / Published online: 17 August 2018

(c) The Author(s) 2018

\begin{abstract}
Purpose Tamoxifen has a wide inter-variability. Recently, two SNPs in the 3'-untranslated region (UTR) of the SULT1A1 gene, rs6839 and rs1042157, have been associated with decreased SULT1A1 activity. The aim of this study is to investigate the role of the rs6839 and rs1042157 on tamoxifen metabolism and relapse-free survival (RFS) in women diagnosed with early-breast cancer receiving tamoxifen.

Methods Samples from 667 patients collected in the CYPTAM study (NTR1509) were used for genotyping (CYP2D6, SULT1A1 rs6839 and rs1042157) and measurements of tamoxifen and metabolites. Patients were categorized in three groups depending on the decreased SULT1A1 activity due to rs6839 and rs1042157: low activity group (rs6839 (GG) and rs1042157 (TT)); high activity group (rs6839 (AA) and rs1042157 (CC)); and medium activity group (all the other combinations of rs6839 and rs1042157). Associations between SULT1A1 phenotypes and clinical outcome (RFS) were explored.

Results In the low SULT1A1 activity group, higher endoxifen and 4-hydroxy-tamoxifen concentrations were found, compared to the medium and high activity group (endoxifen: 31.23 vs. 30.51 vs. $27.00, p$ value: 0.016 ; 4-hydroxy-tamoxifen: 5.55 vs. 5.27 vs. $4.94, p$ value:0.05). In terms of relapse, the low activity group had a borderline better outcome compared to the medium and high SULTIAI activity group (adjusted Hazard ratio: 0.297; 95\% CI 0.088-1.000; $p$ value: 0.05).

Conclusion Our results suggested that rs6839 and rs 1042157 SNPs have a minor effect on the concentrations and metabolic ratios of tamoxifen and its metabolites, and RFS in women receiving adjuvant tamoxifen.
\end{abstract}

Keywords Tamoxifen $\cdot$ Endoxifen $\cdot$ Rs6839 $\cdot$ Rs1042157 $\cdot$ SULT1A1

Electronic supplementary material The online version of this article (https://doi.org/10.1007/s10549-018-4923-7) contains supplementary material, which is available to authorized users.

Henk-Jan Guchelaar

h.j.guchelaar@lumc.nl

1 Department of Clinical Pharmacy \& Toxicology, Leiden University Medical Center, Albinusdreef 2, 2300 RC Leiden, The Netherlands

2 Leiden Network for Personalised Therapeutics, Leiden University Medical Center, Leiden, The Netherlands

3 Department of Medical Oncology, Netherlands Cancer Institute/Antoni van Leeuwenhoek, Amsterdam, The Netherlands

4 Department of Medical Oncology, Leiden University Medical Center, Leiden, The Netherlands

\section{Introduction}

Tamoxifen is commonly used as adjuvant endocrine therapy to treat patients diagnosed with breast cancer $[1,2]$. Being a prodrug, tamoxifen is bioactivated by several cytochrome P-450 enzymes to its primary metabolites, 4-hydroxytamoxifen, and $\mathrm{N}$-desmethyl-tamoxifen (NDM-tamoxifen). Thereafter, conversion into endoxifen takes place (Fig. 1), mainly controlled by CYP2D6, among other enzymes. Around $92 \%$ of tamoxifen metabolism accounts for the biotransformation of tamoxifen into NDM-tamoxifen, whereas the conversion of tamoxifen into 4-hydroxy-tamoxifen only represents $7 \%$ [3].

Both endoxifen and 4-hydroxy-tamoxifen have equal affinity for the estrogen receptor $\alpha$ [4], but endoxifen is considered the most clinically relevant tamoxifen metabolite, since it is found in 5-10 times higher concentrations than 4-hydroxy-tamoxifen [5]. While CYP2D6 is the rate-limiting enzyme in tamoxifen metabolism, it cannot fully explain the 


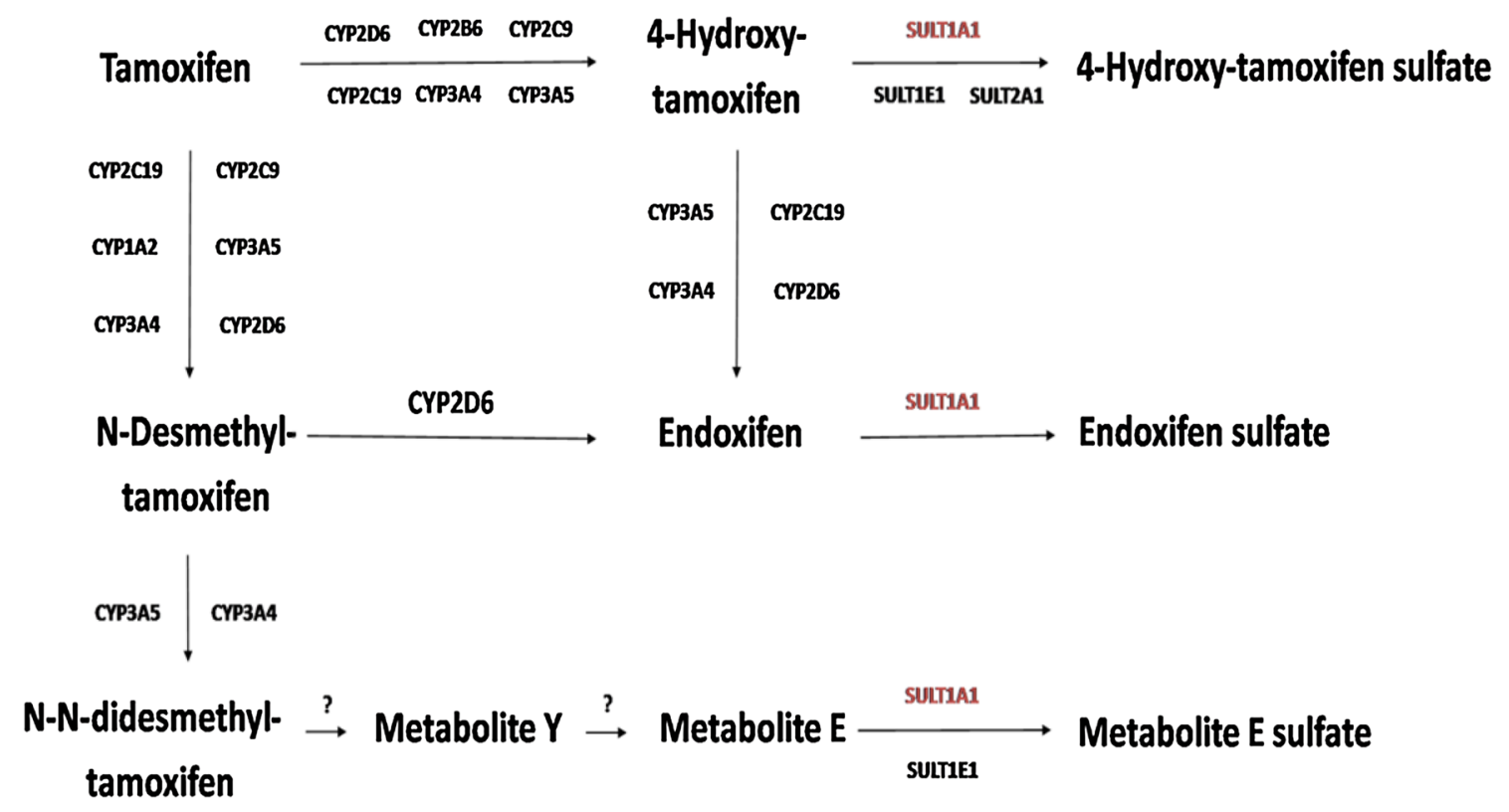

Fig. 1 Tamoxifen metabolism

inter-patient variability of tamoxifen metabolism [6]. Other genetic polymorphisms in different enzymes than CYP2D6 have been suggested to influence tamoxifen metabolism as well [7].

Sulfotransferases (SULTs) are classified as phase II enzymes involved in the biotransformation of a variety of drugs $[7,8]$. By adding a sulfonyl group to xeno- and endobiotics, more hydrophilic molecules are obtained facilitating their renal excretion $[8,9]$. SULT1A1 is the most expressed isoform of the SULT enzymes in the human liver $[10,11]$. In tamoxifen metabolism, SULT1A1 mainly catalyzes the transformation of 4-hydroxy-tamoxifen into inactive 4-hydroxy-tamoxifen sulfate and endoxifen into inactive endoxifen sulfate (Fig. 1). In addition, SULT1A1 is also involved in the inactivation of NDM-tamoxifen, after several consecutive reactions, into Metabolite E sulfate [3, 9, $12,13]$.

Several SULT1A1 Single-Nucleotide Polymorphisms (SNPs) have been described and found associated with clinical outcome in tamoxifen-treated patients. Nowell [14] and Wegman [15] reported that SULTIAI*2/*2 carriers had worse outcome in breast cancer patients treated with tamoxifen compared to both homozygous and heterozygous SULTIAI* 1 carriers. However, studies performed later did not reproduce these results, since no significant associations were found [16-18]. Consequently, the effect of SULTIAI and clinical outcome among tamoxifen-treated patients is still unclear.

SULT1A1 genetic variation and its influence on tamoxifen and its metabolites concentrations and metabolic ratios (MR) have been described. While Jin [19] and
Fernandez-Santander [20] showed no association between SULT1Al genotypes and tamoxifen and its metabolites concentrations, Gjerde and colleagues found an association between SULT1A1 genotype and the metabolic ratios (MR) of NDM-tamoxifen/tamoxifen (Fig. 1) [21].

In the same manner, copy number variation in SULT1Al has been described as a prominent contributor to the intervariability of SULT1A1 enzymatic activity [22]. Hebbring and colleagues reported an in vitro association between CNV and SULT1A1 enzyme activity. The role of SULT1A1 CNVs in tamoxifen efficacy has also been examined, but no significant relationship after 14 years of follow-up between disease-free survival and SULT1A1 CNVs was found [22]. However, this result might be explained by ethnic differences in the enrolled women, who were primarily Caucasian. Indeed, SULT1A1 CNV is most frequently seen in AfricanAmerican individuals, but infrequently occurs in other ethnicities [22].

Recently, two other SULTIAI SNPs, rs6839 and rs 1042157, have been identified and characterized in the 3'-untranslated region (UTR) of the SULTIAl gene [23]. According to the authors, both SNPs are in linkage disequilibrium $\left(D^{\prime}=0.83\right)$ and associated with decreased activity of the SULT1A1 enzymatic activity. To date, only two studies have analyzed the effect of both SNPs and cancer risk [24, 25].

To the best of our knowledge, the role of rs6839 and rs1042157 in tamoxifen metabolism and RFS has not yet been examined. Therefore, the aim of the current study is to explore the role of the rs6839 and rs1042157 SNPs on tamoxifen pharmacokinetics and RFS in the CYPTAM 
cohort of women with early breast cancer using adjuvant tamoxifen [26, 27].

\section{Methods}

Study design and objectives: effect of 3'-UTR of SULT1A1 SNPs on tamoxifen metabolism and clinical outcome.

The CYPTAM study (NTR1509) is a completed prospective clinical study carried out in Belgium and The Netherlands [26]. The aim of this clinical study was to investigate CYP2D6 predicted phenotypes and endoxifen serum concentrations with clinical outcome (relapse-free and diseasefree survival, and overall survival). Briefly, women using tamoxifen at a daily dose of $20 \mathrm{mg}$ as adjuvant endocrine therapy for early breast cancer were asked to participate in this multicenter study. The study protocol of the CYPTAM study was approved by The Medical Ethical Committee of the Leiden University Medical Center (The Netherlands). Written informed consent was obtained from all of the included patients. Pregnancy, breast feeding, and previous malignancy were considered exclusion criteria, with the exception of appropriately treated patients with in-situ cervix carcinoma and basal cell carcinoma. After receiving tamoxifen for a minimum of two months, whole blood and serum samples were collected for genotyping and determination of tamoxifen and its metabolites (NDM-tamoxifen, 4-hydroxy-tamoxifen and endoxifen), respectively.

To investigate the role of rs6839 and rs1042157 SNPs, serum and whole blood samples and clinical data and follow-up from women enrolled in the CYPTAM were readily available for analysis. Since both rs6839 and rs1042157 SNPs are in linkage disequilibrium, groups were required in order to understand the combined effect of both SNPs on tamoxifen metabolism and efficacy. Therefore, three different groups were made according to the known effect of rs6839 and rs1042157 on SULT1A1 enzyme activity. These groups were defined as low, medium, and high SULT1A1 activity groups, as follows: low activity group was defined as the combination of rs6839 (GG) and rs1042157 (TT); high activity group was compound by rs6839 (AA) and rs1042157 (CC); medium activity group was formed by the following combinations: rs6839 (AG) and rs1042157 (CC); rs6839 (AA) and rs1042157 (CT); rs6839 (AG) and rs1042157 (CT); rs6839 (GG) and rs1042157 (CT); rs6839 (AA) and rs1042157 (TT); and rs6839 (AG) and rs1042157 (TT).

The first objective of this pharmacogenetic study was to compare the combined effect of both SNPs on tamoxifen metabolism by comparing differences in endoxifen concentrations and metabolic ratios of tamoxifen and its metabolites (NDM-tamoxifen, 4-hydroxy-tamoxifen, and endoxifen) across the different groups. The secondary objective of this research was to investigate the impact of the 3'- UTR SULT1A1 SNPs groups on tamoxifen efficacy. In the CYPTAM study, the primary endpoint was relapsefree survival (RFS), defined as the time from study enrolment until loco-regional recurrence, second breast cancer, or distant recurrence. If patients switched to an aromatase inhibitor, patients were censored at the time of tamoxifen discontinuation [26].

\section{Tamoxifen and its metabolites measurements}

In order to ensure tamoxifen and metabolite steady-state concentrations, a minimum of two-month treatment with tamoxifen was required before sampling. To adequately assess tamoxifen and its metabolites trough levels, samples were collected at least twelve hours after the last tamoxifen intake.

Concentrations were determined using high-performance liquid chromatography-tandem mass spectrometry (HPLCMS/MS). The bioanalytical assay was developed and validated by the laboratory of Clinical Pharmacy and Toxicology Department at Leiden University Medical Center, and it is a method comparable to another method already reported [28].

\section{Genotyping: CYP2D6, rs6839, and rs1042157}

CYP2D6 genotyping was performed with Amplichip CYP450 test (Roche Diagnostic, Indianapolis, US) to evaluate the major CYP2D6 alleles in DNA previously retrieved from the CYPTAM patients. More detailed information regarding the CYP2D6 genotypes is described elsewhere $[29,30]$. Genotype analysis for rs6839 and rs 1042157 was performed using Pyrosequencing (Qiagen, Venlo, The Netherlands) following standard procedures and the instructions of the manufacturer.

\section{Statistical analysis}

To test linkage disequilibrium between both rs6839 and rs1042157, D' was calculated with Chi-square statistics $\left(\chi^{2}\right)$. Metabolic ratios were defined as concentration of substrate divided by metabolite concentration. ANOVA test was used to compare mean concentration levels and metabolic ratios of tamoxifen and its metabolites (NDM-tamoxifen, 4-hydroy-tamoxifen and endoxifen) between the low, medium, and high SULTIAl activity groups. Multiple linear regression analysis was used to analyze the contributions of rs6839 and rs1042157. By using the base model in which the CYP2D6 status only partly contributes to explaining the total variability of concentrations and metabolic ratios of tamoxifen, endoxifen, 4-hydroxy-tamoxifen, and NDMtamoxifen, these 3'-UTR SULT1A1 rs6839 and rs 1042157 
SNPs were added to the model to investigate their effects on the total variance.

Cox regression analysis was performed to assess whether RFS varied according to the different baseline characteristics across all the groups. If in the univariable analysis, a covariable had a $p$ value below 0.1 , this covariable was fitted in the multivariable model. Due to their clinical importance for the survival outcome in breast cancer patients, tumor and nodal stage, Her2 receptor status, and histological grade and classification were also included in the multivariable analysis, regardless of the results in the univariable analysis. Kaplan-Meier method was used to estimate the distributions of RFS, whereas a log-rank test was performed to compare the clinical outcome with genetic 3'-UTR SULT1A1 rs6839 and rs1042157 SNPs. Statistical analyses were assessed with IBM SPSS for Windows, Version 23.0. In all cases, $p$ values below 0.05 were considered statistically significant.

\section{Results}

\section{Study population}

In the CYPTAM study, 667 women were included in 25 Dutch and Belgian hospitals. More detailed clinical characteristics of the included patients in the core CYPTAM study are reported elsewhere $[30,31]$.

For the purpose of this pharmacogenetic study, three groups with low, medium, and high SULT1A1 activity groups were made. At enrolment, all groups of patients were comparable regarding mean age, tumor and nodal stage, histologic grade and classification, HER2 and progesterone receptor status, type of main surgery (mastectomy or breast conserving surgery) and axillar surgery (sentinel node procedure only or axillary lymph node dissection), adjuvant radiotherapy and chemotherapy, and treatment with trastuzumab ( $p$ value $>0.05$ ). An overview of the baseline characteristics of the enrolled patients by the three groups is listed in Table 1.

\section{Genotype distributions: rs6839 and rs1042157 SNPs}

Genotype distribution for rs 1042157 was consistent with Hardy-Weinberg equilibrium $(\chi 2=2.98, p=0.084)$, while for rs6839 it was found not to be in Hardy-Weinberg equilibrium $(\chi 2=13.44, p=0.00025)$. However, genotype frequencies of rs6839 were similar to allelic frequencies reported previously for the Caucasian population and described on the National Center for Biotechnology Information website (NCBI, http://www.ncbi.nlm.nih.gov). Linkage Disequilibrium was analyzed for both 3'-UTR SULT1A1 variants and a significantly strong association was found for rs6839 and $\operatorname{rs} 1042157\left(D^{\prime}=0.74, p<0.0001\right)$. The variant allele frequencies of rs6839 and 1,042,157 are described in Supplementary Table 1.

\section{Association between tamoxifen and its metabolites and 3'-UTR SULT1A1 groups}

The mean concentration levels of tamoxifen and NDMtamoxifen across the 3'-UTR SULT1A1 groups did not significantly differ $(p>0.05)$. In contrast, endoxifen and 4-hydroxy-tamoxifen mean concentrations in the low activity group were statistically significantly higher, compared to the other groups (endoxifen: $p$ value $=0.016$; 4-hydroxytamoxifen: $p$ value $=0.050$ ). Figure 2 shows the associations comparing low, medium, and high activity groups regarding the mean concentrations and metabolic ratios of tamoxifen and its metabolites. Of note, endoxifen and 4-hydroxytamoxifen concentrations were $15.7 \%$ and $12.3 \%$ higher in the low activity group compared to the high activity group (endoxifen: 31.23 vs. $27.00 \mathrm{nM}$; 4-hydroxy-tamoxifen: 5.55 vs. $4.94 \mathrm{nM}$ ). In Table 2, an overview of the mean concentration levels and metabolic ratios of tamoxifen, endoxifen, 4-hydroyx-tamoxifen, and NDM-tamoxifen is presented.

\section{Clinical outcome and 3'-UTR SULT1A1 groups}

An overall log-rank test comparing the low, medium, and high SULT1A1 activity groups, did not show differences in RFS across the groups, since no statistically significance was obtained ( $p$ value $=0.127$; see Fig. 3). Interestingly, when comparing the low and high activity groups, a statistical difference in RFS was found (Log-rank test: $p$ value $=0.042$; see Fig. 3).

In the same line, the uni- and multivariable Cox regression analyses also found a trend towards better RFS in the low activity group (Adjusted HR:0.297; 95\% CI $0.088-1.000 ; p$ value: 0.05 ; see Table 3 ), compared to the medium and high activity group. A comparison between the extreme groups, low and high SULT1A1 activity, revealed a significantly lower risk for recurrence in the low activity group in both uni- and multivariable Cox regression analyses (Adjusted HR: 0.286; 95\% CI 0.084-0.976; $p$ value: 0.046; see Table 3).

\section{Association of tamoxifen metabolism with rs6839 and rs1042157 SNPs}

Genetic variances in CYP2D6 only partly contribute to explaining the inter-patient variability $\left(R^{2}\right)$ of tamoxifen and its metabolites concentrations and metabolic ratios [29, 32]. When rs6839 and rs 1042157 SNPs were fitted in the model, the inter-patient variability $\left(R^{2}\right)$ of (log-transformed) concentrations and metabolic ratios of tamoxifen and its metabolites increased for all the cases, by 0.4 to $1.3 \%$. Also, 
Table 1 Baseline characteristics of the CYPTAM patients by 3' UTR SULT1A1 high, medium, and low activity groups

\begin{tabular}{|c|c|c|c|c|c|c|c|c|}
\hline & & \multicolumn{6}{|c|}{ 3' UTR SULT1A1 rs6839 and rs1042157 SNPs groups } & \multirow[t]{3}{*}{$p$ value } \\
\hline & & \multicolumn{2}{|c|}{$\begin{array}{l}\text { High activity group } \\
(N=231)\end{array}$} & \multicolumn{2}{|c|}{$\begin{array}{l}\text { Medium activity } \\
\text { group }(N=324)\end{array}$} & \multicolumn{2}{|c|}{$\begin{array}{l}\text { Low activity group } \\
(N=105)\end{array}$} & \\
\hline & & $N$ & $(\%)$ & $N$ & $(\%)$ & $N$ & $(\%)$ & \\
\hline Age at enrolment & Mean in years $(\mathrm{SD})$ & 56.2 & 11.2 & 56.9 & 11.4 & 54.6 & 9.8 & 0.155 \\
\hline \multirow[t]{4}{*}{ Tumor stage } & $\mathrm{T} 1$ & 121 & 52.4 & 170 & 52.5 & 58 & 55.2 & 0.936 \\
\hline & $\mathrm{T} 2$ & 96 & 41.6 & 137 & 42.3 & 41 & 39.0 & \\
\hline & $\mathrm{T} 3 / \mathrm{T} 4$ & 12 & 5.2 & 12 & 3.7 & 4 & 3.8 & \\
\hline & Not specified & 2 & 0.9 & 5 & 1.5 & 2 & 1.9 & \\
\hline \multirow[t]{5}{*}{ Nodal stage } & No & 110 & 47.6 & 158 & 48.8 & 45 & 42.9 & 0.719 \\
\hline & N1 & 92 & 39.8 & 129 & 39.8 & 43 & 41.0 & \\
\hline & $\mathrm{N} 2$ & 19 & 8.2 & 27 & 8.3 & 10 & 9.5 & \\
\hline & N3 & 10 & 4.3 & 8 & 2.5 & 6 & 5.7 & \\
\hline & Not specified & 0 & 0.0 & 2 & 0.6 & 1 & 1.0 & \\
\hline \multirow[t]{4}{*}{ Histological classification } & Ductal adenocarcinoma & 178 & 77.1 & 248 & 76.5 & 78 & 74.3 & 0.738 \\
\hline & Lobular adenocarcinoma & 35 & 15.2 & 42 & 13.0 & 14 & 13.3 & \\
\hline & Other & 18 & 7.8 & 32 & 9.9 & 12 & 11.4 & \\
\hline & Not specified & 0 & 0.0 & 2 & 0.6 & 1 & 1.0 & \\
\hline \multirow[t]{4}{*}{ Histological grade } & G1 & 36 & 15.6 & 42 & 13.0 & 16 & 15.2 & 0.702 \\
\hline & $\mathrm{G} 2$ & 124 & 53.7 & 189 & 58.3 & 61 & 58.1 & \\
\hline & G3 & 70 & 30.3 & 89 & 27.5 & 26 & 24.8 & \\
\hline & Not specified & 1 & 0.4 & 4 & 1.2 & 2 & 1.9 & \\
\hline \multirow[t]{3}{*}{ Progesterone receptor status } & Positive & 186 & 80.5 & 256 & 79.0 & 85 & 81.0 & 0.973 \\
\hline & Negative & 42 & 18.2 & 63 & 19.4 & 18 & 17.1 & \\
\hline & Not specified & 3 & 1.3 & 5 & 1.5 & 2 & 1.9 & \\
\hline \multirow[t]{5}{*}{ HER2 receptor status } & 0 & 135 & 58.4 & 209 & 64.5 & 58 & 55.2 & 0.449 \\
\hline & $1+$ & 68 & 29.4 & 71 & 21.9 & 28 & 26.7 & \\
\hline & $2+$ & 11 & 4.8 & 17 & 5.2 & 7 & 6.7 & \\
\hline & $3+$ & 17 & 7.4 & 25 & 7.7 & 11 & 10.5 & \\
\hline & Not specified & 0 & 0.0 & 2 & 0.6 & 1 & 1.0 & \\
\hline \multirow[t]{3}{*}{ FISH } & Positive (amplification) & 17 & 7.4 & 29 & 9.0 & 11 & 10.5 & 0.584 \\
\hline & Negative & 214 & 92.6 & 293 & 90.4 & 93 & 88.6 & \\
\hline & Not specified & 0 & 0.0 & 2 & 0.6 & 1 & 1.0 & \\
\hline \multirow[t]{3}{*}{ Surgery } & Mastectomy & 116 & 50.2 & 142 & 43.8 & 47 & 44.8 & 0.347 \\
\hline & Breast conserving & 114 & 49.4 & 180 & 55.6 & 56 & 53.3 & \\
\hline & Not specified & 1 & 0.4 & 2 & 0.6 & 2 & 1.9 & \\
\hline \multirow[t]{3}{*}{ Surgery axilla } & Sentinel node procedure only & 110 & 47.6 & 164 & 50.6 & 55 & 52.4 & 0.517 \\
\hline & Axillary lymph node dissection & 120 & 51.9 & 158 & 48.8 & 48 & 45.7 & \\
\hline & Not specified & 1 & 0.4 & 2 & 0.6 & 2 & 1.9 & \\
\hline \multirow[t]{3}{*}{ Adjuvant radiotherapy } & Yes & 156 & 67.5 & 231 & 71.3 & 71 & 67.6 & 0.546 \\
\hline & No & 75 & 32.5 & 91 & 28.1 & 33 & 31.4 & \\
\hline & Not specified & 0 & 0.0 & 2 & 0.6 & 1 & 1.0 & \\
\hline \multirow[t]{3}{*}{ Adjuvant chemotherapy } & Yes & 137 & 59.3 & 198 & 61.1 & 66 & 62.9 & 0.664 \\
\hline & No & 94 & 40.7 & 124 & 38.3 & 38 & 36.2 & \\
\hline & Not specified & 0 & 0.0 & 2 & 0.6 & 1 & 1.0 & \\
\hline \multirow[t]{3}{*}{ Trastuzumab therapy } & Yes & 19 & 8.2 & 28 & 8.6 & 10 & 9.5 & 0.442 \\
\hline & No & 212 & 91.8 & 291 & 89.8 & 94 & 89.5 & \\
\hline & Not specified & 0 & 0.0 & 5 & 1.5 & 1 & 1.0 & \\
\hline
\end{tabular}

3'UTR 3' Untranslated region; SD standard deviation 
the explained variance $\left(R^{2}\right)$ of the (log-transformed) concentrations of endoxifen only marginally improved from 42.3 to $43.6 \%$. An overview of the rs6839 and rs 1042157 covariate analysis is presented in Supplementary Table 2.

\section{Discussion}

This is the first study in which the role of 3'-UTR SULTIAI rs6839 and rs1042157 SNPs on tamoxifen metabolism and clinical outcome in early-breast cancer patients was examined. This study shows that patients with low SULT1A1 activity [rs6839 (GG) and rs1042157 (TT)] reached higher endoxifen and 4-hydroxy-tamoxifen concentration levels, but this small effect did not translate in improved RFS.

SULT1A1 is an important enzyme in tamoxifen elimination and it is involved in two relevant parts of the tamoxifen metabolic pathway: the transformation of 4-hydroxytamoxifen and endoxifen into 4-hydroxy-tamoxifen sulfate and endoxifen sulfate, respectively. As described by Yu and colleagues, 3'UTR SULT1A1 rs6839 and rs1042157 SNPs are associated with a decreased SULT1A1 enzymatic activity, and both SNPs contribute to explaining the variability of SULT1A1 enzyme activity [23]. Based on the results of $\mathrm{Yu}$ and colleagues, we hypothesized that lower SULT1A1 enzymatic activity conferred by the presence of rs6839 and rs1042157 SNPs would translate in higher concentrations of endoxifen and 4-hydroxy-tamoxifen. Our results confirmed this hypothesis, since higher concentrations of both endoxifen and 4-hydroxy-tamoxifen were found.

The transformation from tamoxifen into NDM-tamoxifen represents $92 \%$ of tamoxifen metabolism, while the metabolic conversion from tamoxifen into 4-hydroxy-tamoxifen
Fig. 2 Association with tamoxifen and its metabolites. a Association of tamoxifen, endoxifen, 4-hydroxy-tamoxifen, and NDM-tamoxifen concentration levels by high, medium, and low SULT1A1 enzyme activity groups. b Association of tamoxifen, endoxifen, 4-hydroxytamoxifen, and NDM-tamoxifen metabolic ratios by high, medium, and low SULT1A1 enzyme activity groups

accounts for only $7 \%$ of tamoxifen metabolism [3]. Accordingly, differences in NDM-tamoxifen concentrations would not be as relevant as compared to the other metabolites, whereas small variations in endoxifen and 4-hydroxy-tamoxifen concentrations might be more significant. Our results suggest that the route 4-hydroxy-tamoxifen to endoxifen, might be more important in the presence of a decreased activity of SULT1A1 enzyme, as a consequence of the lower elimination of endoxifen and 4-hydroxy-tamoxifen.

In line with these results, a lower risk for relapse was found in the low activity group, compared to the high activity group. While the increased endoxifen concentration levels and better clinical outcome are completely in line, we feel that this interpretation should be carefully considered, since the association between endoxifen concentration and clinical outcome remains uncertain.

Both endoxifen and 4-hydroxy-tamoxifen have comparable anti-estrogenic activity [4], yet only endoxifen is seen as the most active metabolite of tamoxifen metabolite, since it is found in higher concentrations than 4-hydroxytamoxifen [5]. Therefore, the relationship between endoxifen concentration levels and RFS has been investigated, but different ranges for endoxifen concentration have been proposed. For instance, Madelensky et al. described a $26 \%$ lower chance of relapse for patients with an endoxifen concentration level above $16 \mathrm{nM}(5.97 \mathrm{ng} / \mathrm{ml})$ [33], whereas

Table 2 Overview of mean concentration levels and metabolic ratios of tamoxifen, endoxifen, 4-hydroxy-tamoxifen and NDM-tamoxifen by high, medium and low activity groups

\begin{tabular}{|c|c|c|c|c|c|c|c|c|}
\hline & $\begin{array}{l}\text { Tamoxifen } \\
(\mathrm{nM})(\mathrm{SD})\end{array}$ & $\begin{array}{l}\text { Endoxifen } \\
(\mathrm{nM})(\mathrm{SD})\end{array}$ & $\begin{array}{l}\text { 4-hydroxy- } \\
\text { tamoxifen } \\
(\mathrm{nM})(\mathrm{SD})\end{array}$ & $\begin{array}{l}\text { NDM-Tamox- } \\
\text { ifen }(\mathrm{nM}) \\
\text { (SD) }\end{array}$ & $\begin{array}{l}\text { MR tamox- } \\
\text { ifen/NDM- } \\
\text { tamoxifen } \\
\text { (SD) }\end{array}$ & $\begin{array}{l}\text { MR } \\
\text { tamoxifen/4- } \\
\text { hydroxy- } \\
\text { tamoxifen } \\
\text { (SD) }\end{array}$ & $\begin{array}{l}\text { MR } \\
\text { 4-hydroxy- } \\
\text { tamoxifen/ } \\
\text { endoxifen } \\
\text { (SD) }\end{array}$ & $\begin{array}{l}\text { MR NDM- } \\
\text { tamoxifen / } \\
\text { endoxifen (SD) }\end{array}$ \\
\hline $\begin{array}{l}\text { High activity } \\
\text { group }(N= \\
231)\end{array}$ & $\begin{array}{l}308.20 \\
\quad(113.17)\end{array}$ & $27.00(14.69)$ & $4.94(2.02)$ & $\begin{array}{l}619.54 \\
\quad(231.10)\end{array}$ & $0.51(0.13)$ & $66.82(23.62)$ & $0.21(0.09)$ & $32.74(27.18)$ \\
\hline $\begin{array}{l}\text { Medium activ- } \\
\text { ity group }(N \\
=324)\end{array}$ & $\begin{array}{l}312.12 \\
\quad(128.38)\end{array}$ & $30.51(15.66)$ & $5.27(2.24)$ & $\begin{array}{l}584.42 \\
\quad(224.65)\end{array}$ & $0.54(0.14)$ & $63.94(26.85)$ & $0.19(0.08)$ & $26.04(22.25)$ \\
\hline $\begin{array}{l}\text { Low activity } \\
\text { group }(N= \\
105)\end{array}$ & $\begin{array}{l}319.84 \\
\quad(122.13)\end{array}$ & $31.23(18.29)$ & $5.55(2.78)$ & $\begin{array}{l}621.20 \\
\quad(210.66)\end{array}$ & $0.52(0.13)$ & $64.52(31.77)$ & $0.21(0.10)$ & $30.88(33.42)$ \\
\hline$p$ value & 0.650 & 0.016 & 0.050 & 0.148 & 0.027 & 0.544 & 0.025 & 0.010 \\
\hline
\end{tabular}

$S D$ standard deviation; $M R$ metabolic ratio 

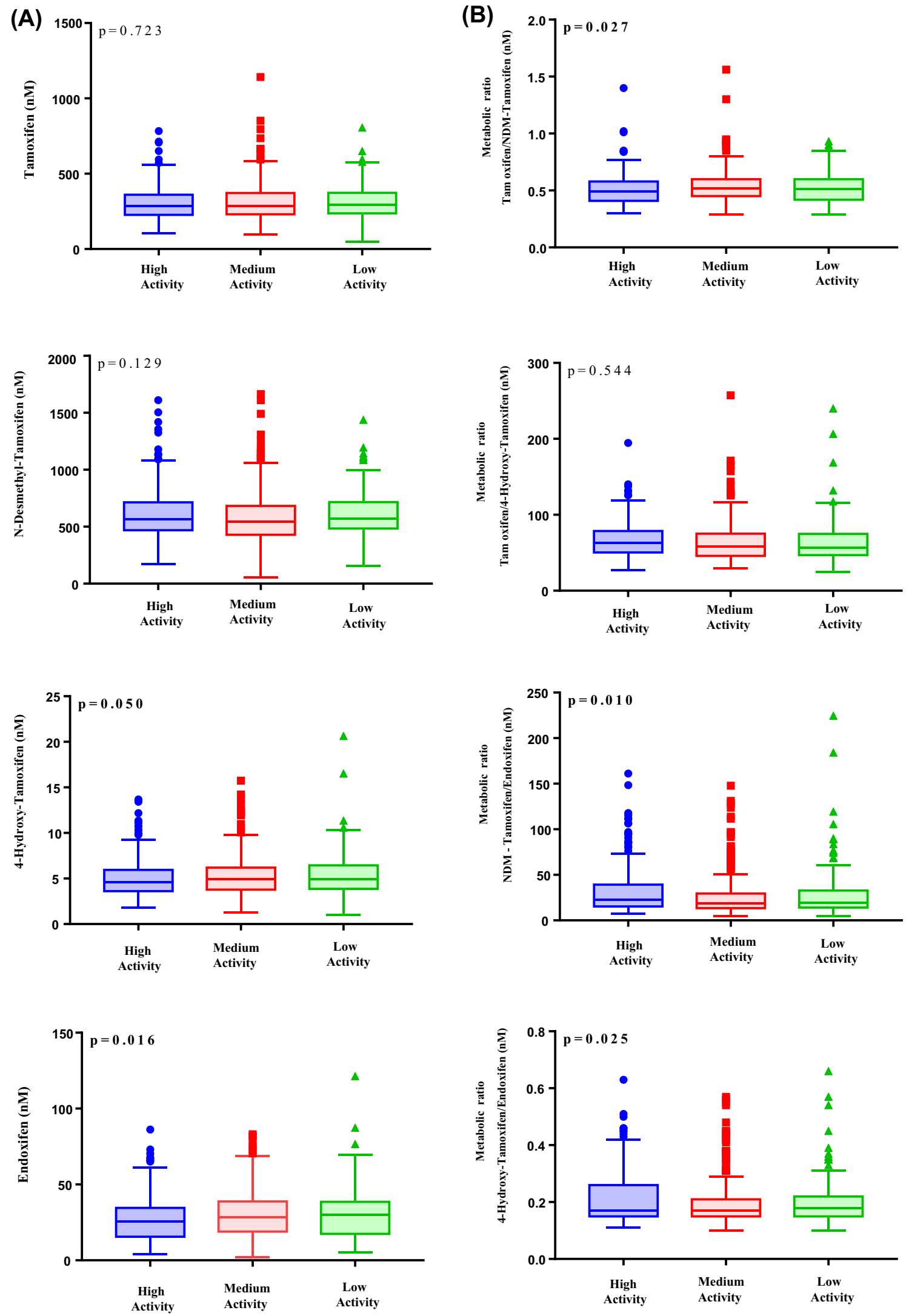
Fig. 3 Kaplan-Meier curve comparing. 3'UTR SULT1A1 rs6839 and rs1042157 SNPs groups. 3'-UTR: 3'untranslated region; SULT1A1: Sulfotransferase $1 \mathrm{~A} 1$

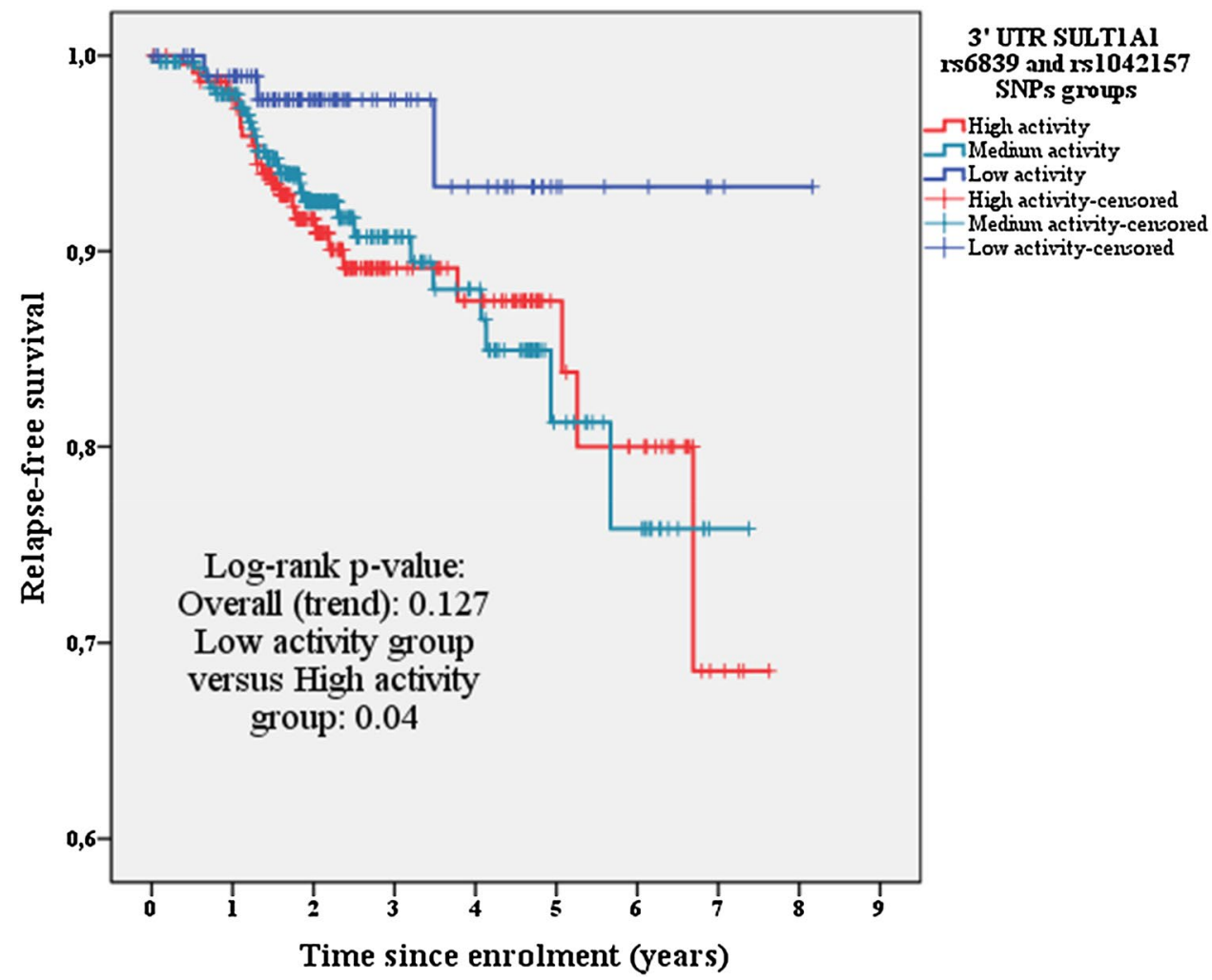

Helland and colleagues reported an even lower limit of $9 \mathrm{nM}(3.36 \mathrm{ng} / \mathrm{ml})$ for better clinical outcomes [34]. In contrast, Neven and colleagues failed to find an association between endoxifen concentration levels and progressionfree survival in the metastatic and neoadjuvant setting [35]. In line with these authors, no association between endoxifen concentration and RFS was found in the core CYPTAM study $[26,27]$. In the present study, a $15.7 \%$ increase of the mean endoxifen serum concentration was found in patients with low SULT1A1 activity, while the explained variance of the concentrations of endoxifen only slightly improved (from 42.3 to $43.6 \%$ ). Accordingly, the combination of the lack of association between endoxifen concentration and RFS in combination with a barely improved explained variance of endoxifen concentrations, it seems unlikely that there is a true association between SULTIAI and RFS caused by the tenue differences in endoxifen concentration levels. Alternative explanations may involve the role of genetic variations in SULT1A1 in breast cancer risk [36] or in endogenous estrogen metabolism [37].
A potential limitation in our analysis might be the fact that rs6839 was not found in HWE. For the pyrosequencing analysis, quality controls were used, and the call-rate in the samples was above $90 \%$, avoiding therefore any technical problem to be reason for this HWE deviation. Also, we performed the pyrosequencing analysis in isolated DNA from whole blood samples. By this way, we prevented any HWE discrepancy due to potential loss of heterozygosity and HWE using tumor material. The rs6839 genotype frequencies were comparable to those reported in the NCBI database [38]. Another possible weakness in our study might be due to the lack of direct measurement of endoxifen sulfate and 4-hydroxy-tamoxifen sulfate levels; instead, we indirectly assessed effects of the SULT1AI SNPs by measuring endoxifen and 4-hydroxy-tamoxifen.

In summary, our results suggest that rs6839 and rs1042157 SNPs have a minor effect on the concentrations and metabolic ratios of tamoxifen and its metabolites, and RFS in women receiving adjuvant tamoxifen, but this impact is not likely to be clinically meaningful. 
Table 3 Cox regression analysis

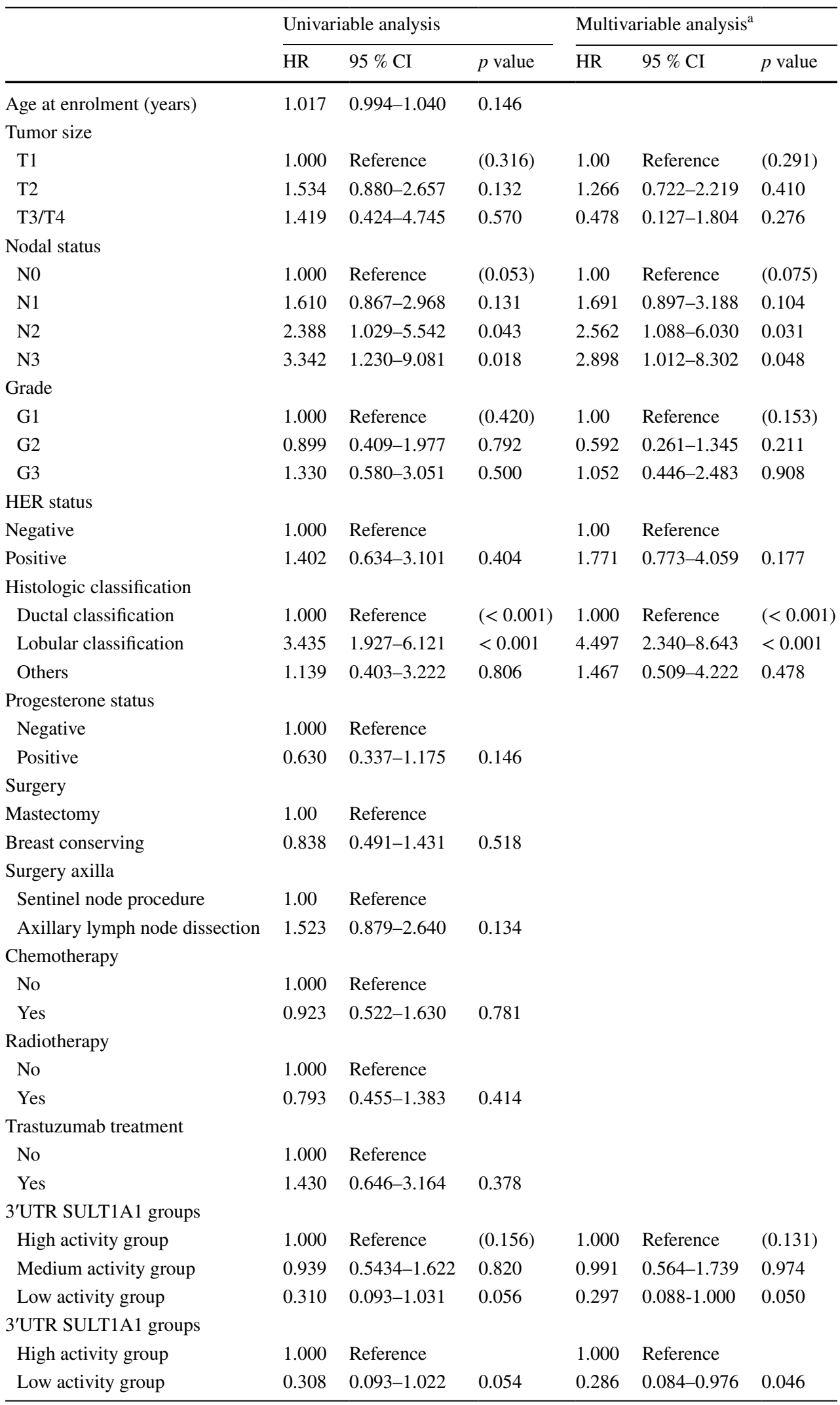

a Adjusted for Her2Neu status, histologic grade and classification, tumor size and nodal stage. 3'UTR 3' untranslated region 
Acknowledgements We are grateful to Roche for kindly providing the Amplichip P450 tests, to the IKNL for data management, and to ZOLEON for its grant.

Author contributions All authors were responsible for writing, critically reviewing, and revising the manuscript, with assistance from a medical writer, and approved the final version prior to submission.

Data Availability The datasets analyzed during the current study are available from the corresponding author on reasonable request.

\section{Compliance with Ethical Standards}

Conflict of interest The authors declare that they have no conflicts of interest.

Ethical Approval All procedures performed in the study described in this manuscript were in accordance with the ethical standards of the institutional and/or national research committee.

Informed Consent Informed consent was obtained from all individual participants included in the study.

Open Access This article is distributed under the terms of the Creative Commons Attribution 4.0 International License (http://creativeco mmons.org/licenses/by/4.0/), which permits unrestricted use, distribution, and reproduction in any medium, provided you give appropriate credit to the original author(s) and the source, provide a link to the Creative Commons license, and indicate if changes were made.

\section{References}

1. Clemons M, Danson S, Howell A (2002) Tamoxifen ("Nolvadex"): a review. Cancer Treat Rev 28(4):165-180

2. Jordan VC (2008) Tamoxifen: catalyst for the change to targeted therapy. Eur J Cancer 44(1):30-38

3. Klein DJ, Thorn CF, Desta Z, Flockhart DA, Altman RB, Klein TE (2013) PharmGKB summary: tamoxifen pathway, pharmacokinetics. Pharmacogenet Genomics 23(11):643-647

4. Johnson MD, Zuo H, Lee KH, Trebley JP, Rae JM, Weatherman RV, Desta Z, Flockhart DA, Skaar TC (2004) Pharmacological characterization of 4-hydroxy-N-desmethyl tamoxifen, a novel active metabolite of tamoxifen. Breast Cancer Res Treat 85(2):151-159

5. Lim YC, Desta Z, Flockhart DA, Skaar TC (2005) Endoxifen (4-hydroxy-N-desmethyl-tamoxifen) has anti-estrogenic effects in breast cancer cells with potency similar to 4-hydroxy-tamoxifen. Cancer Chemother Pharmacol 55(5):471-478

6. Del Re M, Citi V, Crucitta S, Rofi E, Belcari F, van Schaik RH, Danesi R (2016) Pharmacogenetics of CYP2D6 and tamoxifen therapy: light at the end of the tunnel? Pharmacol Res 107:398-406

7. Binkhorst L, Mathijssen RH, Jager A, van Gelder T (2015) Individualization of tamoxifen therapy: much more than just CYP2D6 genotyping. Cancer Treat Rev 41(3):289-299

8. Nowell S, Falany CN (2006) Pharmacogenetics of human cytosolic sulfotransferases. Oncogene 25(11):1673-1678

9. Kiyotani K, Mushiroda T, Nakamura Y, Zembutsu H (2012) Pharmacogenomics of tamoxifen: roles of drug metabolizing enzymes and transporters. Drug Metab Pharmacokinet 27(1):122-131
10. Daniels J, Kadlubar S (2014) Pharmacogenetics of SULT1A1. Pharmacogenomics 15(14):1823-1838

11. Li X, Anderson RJ (1999) Sulfation of iodothyronines by recombinant human liver steroid sulfotransferases. Biochem Biophys Res Commun 263(3):632-639

12. Lu X, Jiang K, Han L, Zhang M, Zhou Y, Ma Y, Zhou Y, Meng S (2015) Sulfonation of curcuminoids: characterization and contribution of individual SULT enzymes. Mol Nutr Food Res 59(4):634-645

13. Desta Z, Ward BA, Soukhova NV, Flockhart DA (2004) Comprehensive evaluation of tamoxifen sequential biotransformation by the human cytochrome $\mathrm{P} 450$ system in vitro: prominent roles for CYP3A and CYP2D6. J Pharmacol Exp Ther 310(3):1062-1075

14. Nowell S, Sweeney C, Winters M, Stone A, Lang NP, Hutchins LF, Kadlubar FF, Ambrosone CB (2002) Association between sulfotransferase 1A1 genotype and survival of breast cancer patients receiving tamoxifen therapy. J Natl Cancer Inst 94(21):1635-1640

15. Wegman P, Elingarami S, Carstensen J, Stal O, Nordenskjold B, Wingren S (2007) Genetic variants of CYP3A5, CYP2D6, SULT1A1, UGT2B15 and tamoxifen response in postmenopausal patients with breast cancer. Breast Cancer Res 9(1):R7

16. Grabinski JL, Smith LS, Chisholm GB, Drengler R, Rodriguez GI, Lang AS, Kalter SP, Garner AM, Fichtel LM, Hollsten J et al (2006) Genotypic and allelic frequencies of SULT1A1 polymorphisms in women receiving adjuvant tamoxifen therapy. Breast Cancer Res Treat 95(1):13-16

17. Serrano D, Lazzeroni M, Zambon CF, Macis D, Maisonneuve $\mathrm{P}$, Johansson H, Guerrieri-Gonzaga A, Plebani M, Basso D, Gjerde $\mathrm{J}$ et al (2011) Efficacy of tamoxifen based on cytochrome P450 2D6, CYP2C19 and SULT1A1 genotype in the Italian Tamoxifen Prevention Trial. Pharmacogenomics J 11(2):100-107

18. Moyer AM, Suman VJ, Weinshilboum RM, Avula R, Black JL, Safgren SL, Kuffel MJ, Ames MM, Ingle JN, Goetz MP (2011) SULT1A1, CYP2C19 and disease-free survival in early breast cancer patients receiving tamoxifen. Pharmacogenomics 12(11):1535-1543

19. Jin Y, Desta Z, Stearns V, Ward B, Ho H, Lee KH, Skaar T, Storniolo AM, Li L, Araba A et al (2005) CYP2D6 genotype, antidepressant use, and tamoxifen metabolism during adjuvant breast cancer treatment. J Natl Cancer Inst 97(1):30-39

20. Fernandez-Santander A, Gaibar M, Novillo A, Romero-Lorca A, Rubio M, Chicharro LM, Tejerina A, Bandres F (2013) Relationship between genotypes Sult1a2 and Cyp2d6 and tamoxifen metabolism in breast cancer patients. PLoS ONE 8(7):e70183

21. Gjerde J, Hauglid M, Breilid H, Lundgren S, Varhaug JE, Kisanga ER, Mellgren G, Steen VM, Lien EA (2008) Effects of CYP2D6 and SULT1A1 genotypes including SULT1A1 gene copy number on tamoxifen metabolism. Ann Oncol 19(1):56-61

22. Hebbring SJ, Moyer AM, Weinshilboum RM (2008) Sulfotransferase gene copy number variation: pharmacogenetics and function. Cytogenet Genome Res 123(1-4):205-210

23. Yu X, Dhakal IB, Beggs M, Edavana VK, Williams S, Zhang X, Mercer K, Ning B, Lang NP, Kadlubar FF et al (2010) Functional genetic variants in the $3^{\prime}$-untranslated region of sulfotransferase isoform 1A1 (SULT1A1) and their effect on enzymatic activity. Toxicol Sci 118(2):391-403

24. Hogervorst JG, van den Brandt PA, Godschalk RW, van Schooten FJ, Schouten LJ (2016) The influence of single nucleotide polymorphisms on the association between dietary acrylamide intake and endometrial cancer risk. Sci Rep 6:34902

25. Ferrucci LM, Cross AJ, Gunter MJ, Ahn J, Mayne ST, Ma X, Chanock SJ, Yeager M, Graubard BI, Berndt SI et al (2010) Xenobiotic metabolizing genes, meat-related exposures, and risk of advanced colorectal adenoma. World Rev Nutr Diet 101:34-45 
26. The CYPTAM study. http://www.trialregister.nl/trialreg/admin/ rctview.asp?TC $=1509$. Accessed 24 May 2018

27. Sanchez-Spitman AB, Dezentjé VO, Swen JJ, Moes DJAR, Batman E, Smorenburg CH, Jongen L, Los M, Guchelaar HJ, Neven P, Gelderblom H (2018) A prospective study on the effect of endoxifen concentration and CYP2D6 phenotypes on clinical outcome in early stage breast cancer patients receiving adjuvant tamoxifen. J Clin Oncol 36(15):523

28. Teunissen SF, Rosing H, Koornstra RHT, Linn SC, Schellens JHM, Schinkel AH, Beijnen JH (2009) Development and validation of a quantitative assay for the analysis of tamoxifen with its four main metabolites and the flavonoids daidzein, genistein and glycitein in human serum using liquid chromatography coupled with tandem mass spectrometry. J Chromatogr B 877(24):2519-2529

29. Dezentje VO, den Hartigh J, Guchelaar H, Hessing T, van der Straaten T, Vletter-Bogaartz JM, Vree R, Maartense E, Smorenburg $\mathrm{CH}$, Putter $\mathrm{H}$ et al: Association between endoxifen serum concentration and predicted CYP2D6 phenotype in a prospective cohort of patients with early-stage breast cancer. J Clin Oncol 2011, 29(15):562

30. Sanchez Spitman AB, Moes D, Gelderblom H, Dezentje VO, Swen JJ, Guchelaar HJ (2017) Effect of CYP3A4*22, CYP3A5*3, and CYP3A combined genotypes on tamoxifen metabolism. Eur J Clin Pharmacol 73(12):1589-1598

31. Sanchez-Spitman AB, Moes DA, Gelderblom H, Dezentje VO, Swen JJ, Guchelaar HJ (2017) The effect of rs5758550 on CYP2D6*2 phenotype and formation of endoxifen in breast cancer patients using tamoxifen. Pharmacogenomics 18(12):1125-1132
32. Dezentje VO, van Schaik RH, Vletter-Bogaartz JM, van der Straaten T, Wessels JA, Kranenbarg EM, Berns EM, Seynaeve C, Putter H, van de Velde CJ et al (2013) CYP2D6 genotype in relation to tamoxifen efficacy in a Dutch cohort of the tamoxifen exemestane adjuvant multinational (TEAM) trial. Breast Cancer Res Treat 140(2):363-373

33. Madlensky L, Natarajan L, Tchu S, Pu M, Mortimer J, Flatt SW, Nikoloff DM, Hillman G, Fontecha MR, Lawrence HJ et al (2011) Tamoxifen metabolite concentrations, CYP2D6 genotype, and breast cancer outcomes. Clin Pharmacol Ther 89(5):718-725

34. Helland T, Henne N, Bifulco E, Naume B, Borgen E, Kristensen VN, Kvaloy JT, Lash TL, Alnaes GIG, van Schaik RH et al (2017) Serum concentrations of active tamoxifen metabolites predict long-term survival in adjuvantly treated breast cancer patients. Breast Cancer Res 19(1):125

35. Neven P, Jongen L, Lintermans A, Van Asten K, Blomme C, Lambrechts D, Poppe A, Wildiers H, Dieudonne AS, Brouckaert O et al (2018) Tamoxifen metabolism and efficacy in breast cancer- a prospective multicentre trial. Clin Cancer Res 24(10):2312-2318

36. Daniels J, Kadlubar S (2013) Sulfotransferase genetic variation: from cancer risk to treatment response. Drug Metab Rev 45(4):415-422

37. Bugano DD, Conforti-Froes N, Yamaguchi NH, Baracat EC (2008) Genetic polymorphisms, the metabolism of estrogens and breast cancer: a review. Eur J Gynaecol Oncol 29(4):313-320

38. Reference SNP Cluster Report: rs6839. https://www.ncbi.nlm.nih. gov/projects/SNP/snp_ref.cgi?rs=6839. Accessed 24 May 2018 